\title{
Readiness for Medical School: a Radical Proposal
}

\author{
Nancy R. Angoff ${ }^{1}$ (D) Michael L. Schwartz ${ }^{1}$
}

Published online: 12 November 2019

(C) International Association of Medical Science Educators 2019

\begin{abstract}
Readiness for medical school, like readiness for reading, includes physiological, psychological, educational, and sociological aspects of growth. Full cognitive and neurodevelopment including higher levels of adult thought order occur generally towards the ends of the third decade. Earlier patient care experiences and an understanding of the social and structural determinants of health demand some experiences in the world. We believe a requirement to prove medical school readiness should be to hold a job for a year that interfaces with the public and proves responsibility, reliability, and accountability as documented by a supervisor.
\end{abstract}

Keywords Medical education $\cdot$ Admissions $\cdot$ Readiness $\cdot$ Neuro development $\cdot$ Cognitive development $\cdot$ Job experience

Much attention is being paid to how residency programs determine graduating medical students are ready to start training in their programs [1]. We believe that more attention should be paid to whether applicants to medical schools are ready to begin the long process of becoming a physician.

A well-known concept in elementary education is reading readiness. It is defined as the time at which a child is capable of learning to read and includes physiological, psychological (emotional and intellectual), educational, and sociological (cultural and environmental) aspects of growth [2]. While a lot has been written about the need for early professional identification in medical training [3, 4], the concept of readiness for that training has not been sufficiently examined. There are several reasons why we should be taking a step back and thinking about the readiness of the students we accept into medical school. Many schools have moved exposure to patients earlier in the curriculum requiring matriculating students to exhibit more developed and advanced interpersonal skills. In addition, we are asking students to understand more deeply the social and structural determinants of health and illness, an

Nancy R. Angoff

Nancy.angoff@yale.edu

Michael L. Schwartz

Michael.schwartz@yale.edu

1 Yale School of Medicine, Office of Education, Harkness D, 367 Cedar Street, New Haven, CT 06510, USA expectation requiring some experience in the world. Students are experiencing earlier burn out, a sign that they may not be up to the emotional demands of the practice of medicine possibly due to biological and developmental reasons in addition to structural reasons. We owe it to our students and to our patients to do a better job of ensuring that students entering medical school are not only intellectually ready, but also emotionally and socially prepared to begin this important journey.

Medical school readiness is the point at which a student is prepared to take on professional identity formation. For different people, that readiness comes at different points in their development. For example, in Robert Kegan's theory of adult development, there are four stages or orders through which people may "transform" in understanding. In the Third order, “...people no longer see others as simply a means to an end; they have internalized one or more systems of meaning (e.g., their family's values, a political or national ideology, a professional or organizational culture)" [5]. This stage is characteristic of adolescence, although many adults may not pass beyond this stage. The Fourth order builds on the understanding of the Third order, but also includes taking on more responsibility for the systems and cultures in which one operates [5] "Indeed, empirical research using Kegan's model supports his prediction that at any particular age, a group of adults will include people at different developmental levels, and also supports his prediction that individuals further along this trajectory are better able to respond to complex work and life challenges," the sorts of challenges inherent in the practice of medicine [6]. 
Many medical students enter school in their early 20s (some before), and may not have reached a level of developmental maturity required of the Third order let alone the Fourth order, and in addition, they may not have a fully developed prefrontal cortex that provides the structural substrate for these cognitive levels. Understanding the biological and behavioral factors that contribute to medical school readiness is becoming increasingly important as medical schools consider curricular reforms that provide earlier and more entrustable exposure to patients in the clinical setting.

Neuroimaging studies have demonstrated that the brain continues to grow and to modify connectivity well into the second decade of life [7, 8]. The tempo of aging is not uniform and varies across areas of the cerebral cortex. Areas responsible for sensory and motor functions mature first while areas of association cortex in the temporal, parietal, and frontal lobes have a more prolonged period of maturation $[9,10]$. Notably, the prefrontal cortex is a region with the most protracted period of growth and maturation expressing significant developmental modifications in volume [11], connectivity [12, 13], gyrification [14], and myelination [15] into the mid 20s. Coincident with this structural maturation of the prefrontal cortex is the maturation and emergence of higher-order cognitive processes, executive functions, and social emotional maturation that are thought to be mediated by the circuitry of this area $[12,14]$. The maturation of these behaviors provides the necessary substrate for the emergence of characteristics of personality and character development that are needed for the professional behaviors required of health care students and professionals. Although the maturation of the prefrontal cortex is largely completed during the third decade of life, variations in the tempo of maturation can be influenced by a number of factors. For example, levels of sex and steroidal hormones, diet, and drug use have been shown to influence myelination and circuit maturation [16]. Thus, individual- and gender-based variations in the maturation of the prefrontal cortex during the period of medical training may influence individual readiness and suggest that curricula must be capable of addressing these variations. As many students begin medical school early in their 20 s, some may not have fully developed higher order cognitive and executive functions that are essential for fully engaging in patient-based care. These may be important considerations when designing curricula that provide early exposure of students to clinical activities with patients.

Currently, as part of the "holistic review" of applicants, we rely on a variety of data to decide on medical school readiness including personal statements, traditional interviews, GPAs and prior school performance, and the MCAT exam [17]. In addition, some schools look for evidence of research and leadership experience and some for service activities. A growing number of medical schools use a standardized structured interview instead of an open-ended conversational interview as a way to assess a student's future performance in medical school. There is concern that traditional interview formats or simulations of educational situations do not accurately predict performance in medical school. Also, when physicians are disciplined later in life by a licensing or regulatory body, a look back at their medical school performance reveals earlier difficulties with the non-cognitive skills, such as interpersonal skills, professionalism, and ethical/moral judgment [18].

In addition to the incomplete neurodevelopmental maturation and the limitations of currently used measures to select appropriate students and to predict readiness for medical school, the premedical course of study can serve to narrow, rather than broaden, students' experiences and focus. For example, those students hoping to complete the typical premedical course of study in 4 years may be less likely to branch out in their course choices to experience the social sciences and humanities. Lloyd B. Minor, the former dean of the medical school at Stanford University wrote, "Through the study of disciplines such as history, philosophy, religion, literature and languages we have unique opportunities to see the world through the eyes of others who have different cultural, social and ethical backgrounds. In so doing, we gain a better appreciation of diversity and ambiguity." [19]. Furthermore, research has shown that "students' exposure to the humanities is linked to important personal qualities and prevention of burnout" [20]. But the premedical curriculum with its emphasis on science and laboratory work can leave little time for a broad exploration of these other disciplines. In addition, with all of the students hoping to enter medical school taking the same premedical courses, there is less opportunity to interact with other students who think differently and are pursuing other goals in life, a type of "pre-med social isolation."

The literature on development of professional identity emphasizes the qualities of caring, compassion, maturity, responsibility, accountability, social and emotional intelligence, structural and cultural sensitivity, and the ability to listen and communicate [3]. Furthermore, more medical schools are accepting their responsibility to a social justice and equity mission that recognizes the health outcomes of our patients which are determined by social, economic, political forces that require attention, understanding, and advocacy. This is a very tall order for a system that has for decades relied on the same grade and test-based methods to identify individuals ready to take on the demands of becoming physicians. Stern and Papadakis state that the best measures of professionalism "capture the behaviors of students in real-world contexts in which they are called on to resolve a professional dilemma that is relevant to their everyday lives." [18].

We suggest a different way to determine readiness of applicants for medical school that would rely on "real world contexts relevant to their everyday lives" to prove that readiness. We propose that premedical students be required to hold a job for at least a year, a job that involves regular hours and 
that interfaces with the public. Examples would be teaching, working in retail, waiting tables. During the course of the year, the student would be required to reflect and write on several experiences. Sample prompts might include the following: If you could have been your supervisor, what changes in the structure of the job, system, would you institute and why? Discuss a situation in which you experienced or witnessed objectional behavior on the part of someone else and what you did or did not do and why. There would be about four of these types of questions that the student would need to write about during the course of the year. In addition, there would be a required letter from the supervisor of the student detailing the student's responsibility, accountability, and contribution to the mission of the job or to the good of other workers. Many students currently take what is called a "gap" year between undergraduate and medical school. What they do with that year, however, is varied and often calculated to meet an algorithm for certain activities believed to be valued by admissions committees. It is not necessarily related to living independently, exposure to different types of people, having to negotiate power structures, and living "in real-world contexts in which they are called on to resolve a professional dilemma that is relevant to their everyday lives" [18].

The process we propose may also impact the tempo and level of maturation of the underlying structural elements of the prefrontal cortex that mediate many of the desired behavioral characteristics of the medical professional. Studies in animal models of cortical development have demonstrated significant effects of enriched social, and peer relationships, as well as stress on prefrontal cortical plasticity and growth [21]. The potential for the enhancement of professional identity characteristics based upon reflection and exposure to relevant social/ work environments is consistent with the notion of experience-expectant development in which the maturing brain undergoes a period during which relevant experiences are required for normal maturation. Furthermore, exposure to real-life experiences and "disorienting dilemmas" [5], allows for transition to a higher developmental stage and readiness to take on the work of responsibility for self and others. A next step would be to study the experience of our students who enter medical school with work experience of the sort we discuss here and compare their course in medical school and beyond with that of students who came right out of college.

Some people may feel that a job for a year is a waste of time for students who need to spend so much time preparing to be physicians. But we suspect this is time incredibly well spent especially if at the end we have students ready to enter medical school with a fully developed prefrontal cortex, mature higher cognitive, emotional, and executive functions with capacity for more sophisticated thinking and reasoning competencies. These students would be better prepared to care for diverse populations of patients in a complex organization and reach the end of medical school ready for the challenges of residency training.

\section{Compliance with Ethical Standards}

Conflict of Interest The authors declare that they have no conflict of interest.

Ethical Approval NA

Informed Consent NA

\section{References}

1. Sklar DP. Matchmaker, matchmaker, make me a match: is there a better way? Acad Med. 2019;94(3):295-7.

2. Smith DE, Chapel JR. Reading readiness. In Reading Horizons: a Journal of Literacy and Language Arts. 1970. https://scholarworks. wmich.edu/reading_horizons/vol10/iss2/3. Accessed 1 Oct 2019.

3. Cruess RL, Cruess SR, Boudreau JD, Snell L, Steinert Y. Reframing medical education to support professional identity formation. Acad Med. 2014;89(11):1446-51.

4. Sharpless J, Baldwin N, Cook R, Kofman A, Morley-Fletcher A, Slotkin R, et al. The becoming: students' reflections on the process of professional identity formation in medical education. Acad Med. 2015;90(6):713-7.

5. Berger JG. A summary of the constructive-developmental theory of Robert Kegan. In Studylib.net. 2007. https://studylib.net/doc/ 7860146/a-summary-of-the-constructive-developmental-theory-ofrobert. .

6. Lewin LO, McManamon A, Stein MTO, Chen DT. Minding the form that transforms: using Kegan's model of adult development to understand personal and professional identity formation in medicine. Acad Med. 2019.

7. Sowell ER, Thompson PM, Holmes CJ, Jernigan TL, Toga AW. In vivo evidence for post-adolescent brain maturation in frontal and striatal regions. Nat Neurosci. 1999;2(10):859-61.

8. Mu SH, Xu M, Duan JX, Zhang J, Tan LH. Localizing age-related changes in brain structure using voxel-based morphometry. Neural Plast. 2017.

9. Johnson SB, Blum RW, Giedd JN. Adolescent maturity and the brain: the promise and pitfalls of neuroscience research in adolescent health policy. J Adolesc Health. 2009;45(3):216-21.

10. Sowell ER, Thompson PM, Tessner KD, Toga AW. Mapping continued brain growth and gray matter density reduction in dorsal frontal cortex: inverse relationships during postadolescent brain maturation. J Neurosci. 2001;21(22):8819-29.

11. Tamnes CK, Herting MM, Goddings AL, Meuwese R, Blakemore SJ, Dahl RE, et al. Development of the cerebral cortex across adolescence: a multisample study of inter-related longitudinal changes in cortical volume, surface area, and thickness. J Neurosci. 2017;37(12):3402-12.

12. Achterberg M, Peper JS, van Duijvenvoorde AC, Mandl RC, Crone EA. Frontostriatal white matter integrity predicts development of delay of gratification: a longitudinal study. J Neurosci. 2016;36(6): 1954-61.

13. Petanjek Z, Judas M, Simic G, Rasin MR, Uylings HB, Rakic P, et al. Extraordinary neoteny of synaptic spines in the human prefrontal cortex. Proc Natl Acad Sci U S A. 2011;108(32):13281-6.

14. Chung YS, Hyatt CJ, Stevens MC. Adolescent maturation of the relationship between cortical gyrification and cognitive ability. Neuroimage. 2017;158:319-31.

15. Giedd JN. Structural magnetic resonance imaging of the adolescent brain. Ann N Y Acad Sci. 2004;1021:77-85.

16. Silvers JA, Insel C, Powers A, Franz P, Helion C, Martin RE, et al. V1PFC-vmPFC-amygdala interactions underlie age-related 
differences in cognitive regulation of emotion. Cereb Cortex. 2017;27(7):3502-14.

17. Association of American Medical Colleges. AAMC Holistic Review Project Retrieved from: https://www.aamc.org/download/ 358700/data/hrp2-pager.pdf.

18. Stern DT, Papadakis M. The developing physician-becoming a professional. N Engl J Med. 2006;355(17):1794-9.

19. Lloyd B. Minor M. The humanities and medicine. In The Stanford Dailey 2014. https:/www.stanforddaily.com/2014/04/06/thehumanities-and-medicine. Accessed 1 Oct 2019.
20. Mangione S, Chakraborti C, Staltari G, Harrison R, Tunkel AR, Liou KT, et al. Medical students' exposure to the humanities correlates with positive personal qualities and reduced burnout: a multiinstitutional U.S. survey. J Gen Intern Med. 2018;33(5):628-34.

21. Kolb B, Mychasiuk R, Muhammad A, Li Y, Frost DO, Gibb R. Experience and the developing prefrontal cortex. Proc Natl Acad Sci U S A. 2012;109(Suppl 2):17186-93.

Publisher's Note Springer Nature remains neutral with regard to jurisdictional claims in published maps and institutional affiliations. 